\title{
TALLER VIRTUAL PARA EL ESTíMULO DEL PENSAMIENTO FILOSÓFICO, MEDIANTE PROMOCIÓN DE LECTURA LITERARIA EN UNIVERSITARIOS
}

\section{A VIRTUAL WORKSHOP TO STIMULATE THE PHILOSOPHICAL THINKING THROUGH LITERARY READING IN UNIVERSITY STUDENTS.}

\author{
Herlinda Flores Badillo ${ }^{1}$ \\ Iván Luengas Palacios² \\ Universidad Veracruzana, México
}

$1 \quad$ Flores es profesora de Tiempo Completo (TC) para el Centro de Idiomas Córdoba (CIC), con veinte años de antigüedad, es profesora perfil PRODEP, fue coordinadora del CIC y actualmente coordina la Especialización en Promoción de la Lectura (EPL), pertenece también al núcleo básico de la Maestría, MEILE. hflores@uv.mx. ORCID: https://orcid.org/0000-0002-1092-6195 https://scholar. google.es/citations?hl=es\&user=AzzFLNgAAAAJ

$2 \quad$ Luengas es profesor, con una licenciatura y una maestría en filosofía, actualmente es estudiante de la Especialización en Promoción de la Lectura de la Universidad Veracruzana. Se ha desempeñado como catedrático y coordinador de eventos académico-culturales en el Centro de Estudios Superiores "Guillermo Nicolás" y en otras instituciones de nivel medio-superior y superior. Originario de Orizaba, Veracruz (México). Tel. 2721546388 Email: ivanluengas88@gmail.com ORCID: https://orcid.org/00000002-8409-9866

\section{RESUMEN}

El presente artículo reporta los hallazgos de un taller virtual de promoción de lectura literaria en una comunidad universitaria de filosofía. El propósito de éste fue promocionar la lectura de obras literarias por placer para estimular el quehacer filosófico, a partir de tópicos existenciales. La situación inicial del grupo intervenido mostró que algunos participantes solo practicaban la lectura utilitaria y que los estudios de filosofía no eran su interés primordial. De igual manera, que éstos consideraban que los textos filosóficos resultaban difíciles de abordar. 
Esto exigió la incorporación de instrumentos que complementaran el gusto por la lectura con la actividad filosófica. Los resultados apuntaron que se pudo tomar conciencia de la importancia de la lectura por placer en los estudios académicos, se favoreció la interpretación inferencial (preámbulo del quehacer filosófico), se suscitó el interés por la filosofía y se motivó a la práctica de la lectura mediante una tertulia literaria dialógica.

\section{PALABRAS CLAVE}

Lectura literaria, quehacer filosófico, temas existenciales, tertulia literaria dialógica, lectura utilitaria, lectura por placer.

\section{ABSTRACT}

This article reports the findings obtained from an online reading promotion workshop for university students of philosophy. The purpose of this intervention was to promote reading literary pieces of work for pleasure to stimulate the philosophical thinking practice by reading existential topics. The initial context of this specific group showed that there were some students who just did utilitarian reading and that philosophy studies were not of their main interests. Furthermore, these learners considered philosophical texts difficult to read. This situation demanded to implement instruments that could complement the philosophical tasks with the pleasure to read. The outcomes revealed that most of the participants were more aware of the importance of reading for pleasure while performing the academic process, their inferential interpretation was favoured (preamble to the philosophical practice), their philosophical interest was arosed and they were motivated to have dialogic literary circles.

\section{KEY WORDS}

Literary reading, philosophical practice, existential topics, dialogic literary circles, utilitarian reading, reading for pleasure.

\section{INTRODUCCIÓN}

El ejercicio de la lectura es una actividad esencial para el desarrollo humano, potencializarla y promoverla en los ambientes educativos, sin lugar a dudas genera mejores estudiantes y prepara futuros ciudadanos con herramientas críticas mejor consolidadas. Sin embargo, en las últimas décadas, la lectura se ha escolarizado demasiado que sólo se la concibe como un medio para el estudio y el aprendizaje, de ahí que "Para algunos profesores y padres de familia la lectura que no tiene el estudio como fin es una distracción, un desperdicio" (Garrido, 2012, p. 19). Esta concepción ha provocado que talleres, cursos y programas de lectura queden condicionados a los estándares academicistas, donde se impone un discurso hegemónico culturalista de ésta, el cual sin pretender contrarrestar sus efectos positivos, termina por asfixiar el gusto por la misma (Sardi, 2014).

Esta situación, entre otras, ha provocado que en México aún se esté lejos de alcanzar un nivel óptimo en el ejercicio de la lectura. Al respecto la prueba internacional PISA (OCDE, 2019) señaló que este país está por debajo del promedio de naciones de la OCDE en lectura, obteniendo 420 puntos de un total de 600 , siendo el promedio 487. Por su parte la prueba nacional PLANEA (Plan Nacional para la Evaluación de los Aprendizajes, 2017) aplicada en el nivel medio-superior y cuya importancia para este estudio radica en que evalúa a los estudiantes que están próximos a ingresar a la universidad, refirió que en lenguaje y comunicación sólo el $9.2 \%$ de los alumnos alcanzó el dominio sobresaliente, el $28.7 \%$ el satisfactorio, el $28.1 \%$ el básico y el $33.9 \%$ el insuficiente. En este último los estudiantes no pueden formular 
inferencias en los textos que leen ni comprender los que son extensos y complejos. Por su parte, el Instituto Nacional de Estadística y Geografía (INEGI, 2020) a través del Módulo sobre Lectura (MOLEC) refirió que 4 de cada 10 personas declararon leer al menos un libro en los últimos doce meses y que el promedio de ejemplares leídos fue de 3.4. De estos que declararon ser lectores, se destaca que sólo el $38.7 \%$ leen por entretenimiento y el $27.1 \%$ por trabajo o estudio, los demás lo hacen por otros motivos.

Como se puede constatar el panorama en el ejercicio de la lectura en México no es alentador, lo cual obedece a diversas fallas en el sistema educativo. Particularmente, en el ámbito universitario, el ejercicio de la lectura se ve desfavorecido por diversas problemáticas. Argüelles (2015) refiere que una de las razones es que la universidad se ha preocupado por impartir conocimientos privilegiando los del área técnica y ha demeritado la formación humanista. Esto ha provocado que las nuevas generaciones tengan aversión a la lectura y desconozcan sus beneficios. A lo sumo la comunidad estudiantil de esta etapa escolar lee para aprobar exámenes y obtener títulos académicos sin asimilar los contenidos cognitivos ni comprenderlos. Por su parte Carlino (2005) señala que es cuestionable la disociación que comúnmente se hace entre el aprendizaje proporcionado en cada disciplina y la lecto-escritura, debido a que éstas se complementan y permiten el desarrollo de otras habilidades. Además, Cassany et al., (2008) considera criticable el que en la universidad se conciban la lectura y la escritura como habilidades uniformes en todos los estudiantes, desvinculadas de lo afectivo, social y personal. Tal concepción ha acarreado estandarizaciones insuficientes que distan de favorecer la comprensión de textos propios de cada disciplina.
Esta forma de entender la enseñanza ha acarreado una serie de problemáticas que van desde conocer sólo generalidades sobre un tema sin ahondar en sus fundamentos hasta conocer sólo un campo específico y perder el sentido de totalidad. De este modo se ha propiciado que la mayor parte del estudiantado no sea capaz de amar el conocimiento en sí mismo, sino sólo con fines utilitarios y económicos. A esto se suma la idea de que el aprendizaje sólo se logra mediante el esfuerzo y no por el regocijo impulsado a través de la lectura por placer (Mata, 2008). Por ello la lectura en la universidad debe poner en contacto directo con los autores para provocar que los universitarios se apasionen y descubran un horizonte más amplio del que el sistema vigente les presenta.

Desde estas consideraciones se constata que todas estas deficiencias se sintetizan en el analfabetismo funcional, el cual refiere que los estudiantes no son capaces de desarrollar sus habilidades de análisis, crítica y expresión de ideas, a pesar de saber leer y escribir, dando como consecuencia una deficiente comprensión lectora y serias limitantes en el desarrollo de la escritura (Morón Arteaga, 2015). De esta problemática se deriva el hecho de que los estudiantes no desarrollen el gusto por la lectura y carezcan de las competencias suficientes para responder a los retos que el mundo globalizado ha traído en la cultura occidental.

\section{PROBLEMÁTICA Y CONTEXTO DE LA INTERVENCIÓN.}

El ejercicio de la lectura es una herramienta fundamental en el estudio de la filosofía, ya que desarrolla las habilidades cognitivas y analíticas necesarias para exponer las ideas con orden y rigor lógico. Sin embargo, quienes acceden a cursar una licenciatura en filosofía no siempre poseen las herramientas académicas suficientes para hacer frente a los contenidos que esta ciencia posee. A partir de una entrevista personalizada sobre la 
práctica de la lectura en el Centro de Estudios Superiores "Guillermo Nicolás" (en lo sucesivo se utilizarán las siglas CESGN), algunos profesores del mismo, señalaron que en diversas generaciones de estudiantes sobresalían las siguientes deficiencias: falta de hábito lector, poca comprensión lectora y dificultad para leer en voz alta. Estas insuficiencias se reflejaban en la incapacidad de algunos estudiantes para identificar la idea central de un texto y sus puntos nodales, o bien para forjarse una opinión crítica y fundamentada. Tales problemáticas han obedecido a diversos factores como la carente formación lectora en las etapas escolares previas en las que se acentúa la lectura utilitaria; la falta de interés por la filosofía, debido a que algunos estudiantes la toman como requisito para continuar hacia los estudios teológicos; y la falta de un programa de lectura que parta de las necesidades de los estudiantes y potencialice el gusto por el conocimiento. Esto ha traído como consecuencia la realización de una lectura meramente utilitaria de obras filosóficas, incluso fragmentaria, lo que no ha permitido una profundización en los tópicos relevantes de la filosofía, ocasionando que algunos estudiantes sólo lean para aprobar materias o entregar trabajos académicos y pierdan la oportunidad de incrementar su capacidad reflexiva, desembocando en el problema del analfabetismo funcional universitario.

Es así que con el fin de evitar los efectos de tal problemática en el CESGN ubicado en la ciudad de Ixtaczoquitlán, Veracruz, se realizó un diagnóstico a un grupo de 17 estudiantes de primer semestre, para conocer su situación lectora e interés por los estudios filosóficos $y$, de esta manera, poder contribuir en su formación lectora y filosófica. De este estudio se pudo conocer que el grupo se conformaba de participantes que provenían de distintas zonas geográficas (indígena, rural y urbana), cuyas edades fluctuaban entre los 18 y 27 años. En cuanto a su situación lectora, el estudio mostró que 6 estudiantes se consideraban lectores utilitarios, 9 lectores autónomos y 2 lectores letrados, siguiendo la clasificación de los tipos de lector de Garrido (2014). También que el grupo contaba con un nivel de lectura satisfactorio, pues el $52.94 \%$ declaró leer al menos una vez a la semana y el $23.53 \%$, más de una vez a la semana, lo cual estuvo en consonancia con el número de lectores autónomos y letrados que juntos sumaban 11 participantes. Sin embargo, se constataron otros datos que llamaron la atención y que se vislumbraron como factores que podrían posibilitar en lo sucesivo una falta de interés por los estudios filosóficos. El primero es que 6 estudiantes veían los estudios de filosofía como una preparación para el estudio de la teología y/o formación sacerdotal. A esto se sumó el que el $41.18 \%$ viera los estudios de filosofía como un medio para incrementar sus conocimientos, es decir, como instrumento de cultura general. Esto reflejó que la actividad filosófica no era el fin primordial, tanto en uno como en otro caso. Además los resultados señalaron que el $52.95 \%$ consideraba que los textos filosóficos eran difíciles de interpretar y el $47.05 \%$ consideró que eran difíciles de leer por sus tecnicismos. De esta manera se llegó a la conclusión de que si no se potenciaba la lectura por placer en los lectores utilitarios y no se estimulaba el quehacer filosófico en el grupo en general, existía el riesgo de incurrir en el analfabetismo funcional universitario.

En cuanto a los temas que a los participantes les interesaba conocer mediante la lectura, fue relevante saber que había una preponderancia de los temas filosóficos, los históricos y los mitológicos. Esto motivó a reafirmar la pertinencia de temas existenciales en el taller y a reconocer este punto como un área de oportunidad más. No obstante, cuando se preguntó sobre el tipo de libros preferidos para realizar lectura por placer, hubo una variante en la que aparecieron en primer lugar los de contenido religioso con el $26.92 \%$, después los 
de filosofía con el $23.08 \%$ y, en tercer lugar, los de literatura con el $19.23 \%$. El porcentaje restante se repartió entre libros de ciencia ficción, científicos e históricos. Este cambio confirmó la tendencia humanista predominante en el grupo intervenido y, a su vez, el contexto religioso de 15 participantes.

\section{METODOLOGÍA}

De modo general, el estudio sigue los lineamientos de la investigación acción, debido a que buscó incidir en un sector de la sociedad mediante una toma de conciencia para transformar su realidad, siendo los destinatarios los protagonistas de tal cambio (Hernández Sampieri et al., 2018). Particularmente, el estudio pretendió medir el impacto que el taller tuvo en los participantes para constatar si éstos pudieron reconocer la importancia de la lectura por placer en los estudios filosóficos, además para mostrar si éstos pudieron alcanzar interpretaciones inferenciales con los recursos proporcionados en el mismo. También se destaca que el taller estuvo inspirado en un modelo hermenéutico y constructivista, debido a que buscó favorecer la interpretación inferencial en la que el lector pudiera mostrar la comprensión de sí mismo y de su entorno (Grondin, 2008). Además, porque los recursos utilizados rescataron el valor de los conocimientos previos eimpulsaron lageneración de sus propias reflexiones o conocimientos (Carretero, 1993). Para cumplir tales propósitos, la cartografía lectora seleccionada tocó algunos temas existenciales como el dolor, la muerte, el sentido de la vida, la libertad y el amor, con la intención de estimular la reflexión filosófica de los participantes, a partir del diálogo. Éste, según Bajtín, permite salir de un lenguaje unificado y estimula una comunicación fundada en la diversidad (Sisto, 2015). Por esta razón, el compartir dialógico fue esencial durante toda la intervención, la cual comprendió 22 sesiones de 50 minutos repartidas en 8 semanas en las que se utilizaron diversos recursos como videos, poemas, novelas y canciones, así como estrategias de lectura grupal e individual, en voz alta, en silencio y dramatizada para disponer a los participantes al ejercicio de la lectura y a la reflexión filosófica.

Respecto a los instrumentos de evaluación, se utilizó inicialmente un cuestionario diagnóstico para conocer la situación lectora, las preferencias de lectura, las motivaciones y dificultades para el estudio de la filosofía. En la parte final se llevó a efecto una entrevista personalizada para verificar el logro de los objetivos y una rúbrica para evaluar el contenido de los ensayos. Además de ello, hubo la elaboración de productos por parte de los participantes que fueron referentes para medir el impacto de los textos utilizados, las estrategias de promoción de la lectura y la capacidad para realizar interpretaciones inferenciales de los textos. Estos productos fueron dos bitácoras de experiencias lectoras realizadas a partir de dos textos literarios y un ensayo filosófico fruto de la implementación de una tertulia literaria dialógica.

\section{DESARROLLO DEL TALLER}

El taller estuvo estructurado en tres partes: la primera fue motivacional, la segunda de estrategias de lectura y la tercera incluyó la implementación de una tertulia literaria dialógica. La primera, realizada en 5 sesiones, tuvo el propósito de favorecer una toma de conciencia sobre la importancia de la lectura por placer e incentivar su práctica. Para ello se utilizaron los siguientes recursos: la lectura gratuita voluntaria que implicó leer en voz alta el fragmento de un texto al inicio de una sesión y ofrecerla a los demás participantes sin esperar comentario alguno; un video de promoción de lectura del escritor Benito Taibo (2020); el poema 20 de 
Pablo Neruda; los artículos de Guardiola (2018) y Cárdenas Guzmán (2018) y 3 escritos que relataron la experiencia personal de iniciación en la lectura por parte del coordinador del taller y algunos participantes voluntarios.

La segunda parte se efectuó en 9 sesiones y tuvo como propósito potenciar el gusto por la lectura con estrategias específicas y la interpretación inferencial. Para ello se continuó con la lectura gratuita y una experiencia personal más de iniciación en ella, compartidas por algunos participantes. A su vez, se propusieron dos textos introductorios para disponer al grupo a la lectura dramatizada: el epígrafe de Rayuela (Cortazar, 2008) y la letra de la canción El campamento de Luis Pescetti. Ambos se practicaron con lectura convencional y luego con lectura dramatizada para constatar la variación de sentido de los textos y favorecer la comprensión, particularmente del primero. Esto permitió corroborar que "[...] la lectura siempre se produce en múltiples direcciones y de maneras impensadas e inadvertidas" (Sardi, 2014, p. 68).

Como núcleo central de esta parte se analizaron dos textos literarios, El banquete (Platón, 1998) y Hamlet (Shakespeare, 2006), los cuales fueron leídos con dos estrategias de lectura: lectura grupal y lectura dramatizada, respectivamente. Posteriormente se analizaron con el apoyo de dos bitácoras de experiencias lectoras (ver Tabla 1), las cuales se trabajaron del siguiente modo: primeramente, se pidió a los participantes contestar la sección de "conocimientos previos" que contenía una pregunta, en el caso de la bitácora 1; y un caso para discutir en grupo respecto a la bitácora 2. Con ello se pretendió introducir al texto a los participantes y rescatar sus conocimientos previos, siguiendo el modelo constructivista. Después se hizo la lectura de algunos apartados previamente seleccionados de dichos libros. Ambos se leyeron en forma grupal y de manera dramatizada sólo en el caso de Hamlet, en el que se repartieron los personajes a los participantes. Al término de las lecturas grupales se hizo un plenario para extraer las ideas principales del texto y contestar de forma individual la columna de "reconstrucción de la trama". Posteriormente se pidió a los participantes, en el caso de El banquete, retomar una frase o idea que fuese significativa y elaborar una reflexión personal en la sección "aprendizaje significativo" de la bitácora de experiencia lectora. En el texto de Hamlet se proporcionaron dos binomios temáticos (locura-amor y venganza-muerte) y se solicitó a los participantes realizar una reflexión al respecto. En esta última sección se pretendió conocer en qué medida los participantes alcanzaron una interpretación inferencial, siguiendo el modelo hermenéutico.

Una vez contestadas las bitácoras se dedicó una sesión para cada texto, en el que se compartieron por parte de algunos participantes las reflexiones personales puestas en las mismas. Al término de cada participación el coordinador retomó algún dato significativo y preguntó por qué se compartió tal dato, con el fin de que el participante profundizara más en el hecho y lo vinculara con una situación de vida. 
Tabla 1

Ejemplo de bitácora de experiencias lectoras

\section{Nombre: \\ Fecha:}

Instrucción: Antes de leer el texto contesta la pregunta de la sección "conocimientos previos". Después participa en la lectura en voz alta del texto y comparte tus comentarios en plenario, apoyándote en las otras dos secciones.

Asigna un tema breve al texto leído:

\section{Conocimientos previos}

¿Qué estrategia ocuparían para descubrir al autor de un asesinato

de un miembro de su familia, teniendo sólo como certeza una fuerte corazonada? Considera que el asesino es alguien con el que convives.

\section{Reconstrucción de la trama}

[Escribe las ideas principales del texto]

\section{Aprendizaje significativo}

A partir de una frase significativa para ti, elabora una reflexión.

Nota. La bitácora fue un instrumento que permitió, por un lado, distinguir en los participantes las ideas que más impactaron del texto en ellos y, por otro, su capacidad para extraer interpretaciones inferenciales y estructurar sus ideas. Con ellas se pudo constatar que los participantes siguieron reflexiones encausadas por rumbos diferentes.

La última parte del taller, realizada en 8 sesiones, se enfocó a la implementación de una tertulia literaria dialógica. Esto con el fin de ampliar el horizonte de comprensión de los textos y generar un ambiente de diálogo entre los participantes que sustituyera los riesgos de una participación competitiva fundada en interacciones de poder (Pulido et al., 2010, p. 299). Para llevarla a efecto se formaron dos equipos seleccionados en base al diagnóstico. En el primero se incluyeron seis participantes autodenominados lectores utilitarios y dos autónomos; en el segundo siete lectores autónomos y dos letrados. Esto como medida estratégica para dar la oportunidad de que todos participaran. Se asignó un día a la semana para llevar a efecto la tertulia de cada grupo, con el fin de dar tiempo suficiente a los participantes en la lectura de los textos escogidos. Estos fueron $E I$ hombre en busca de sentido (Frankl, 1991) para el grupo de lectores utilitarios y autónomos, y $L a$ peste (Camus, 2019) para el grupo de lectores letrados y autónomos.

Antes de iniciar las tertulias se hizo una presentación a los participantes para que conocieran en qué consisten, cuáles son sus principios y cómo funcionan. Después se pasó a comentar la parte seleccionada del texto por cada participante. Se les pidió que señalaran el párrafo que les interesó, que lo leyeran en voz alta y que dijeran por qué fue significativo. En la primera y segunda reunión la participación fue un tanto mecánica, pero a partir de la tercera se hizo más interactiva y espontánea. De esta forma y para efectos de la conclusión del taller se hicieron tres tertulias para cada grupo, con el fin de identificar los temas existenciales contenidos en las obras y adquirir algunos elementos para la realización de un ensayo filosófico, con el cual se terminó la evaluación del taller. 


\section{PRINCIPALES PROBLEMÁTICAS PRESENTADAS A LO LARGO DE LA INTERVENCIÓN}

Fallas de conexión en internet. Los problemas de conexión afectaron el desarrollo adecuado del taller en dos aspectos: participación y asistencia. Respecto al primero se destaca que éste se vio desfavorecido, una vez que los estudiantes que pertenecían a zonas indígenas o rurales, no siempre podían externar sus comentarios o activar algunas funciones de la plataforma, como el compartir pantalla o el uso de la cámara. De igual forma la asistencia se vio afectada alcanzando sólo el $82.97 \%$, debido a que algunos estudiantes no pudieron conectarse a todas las sesiones o sólo podían conectarse por lapsos discontinuos. Ocasionalmente los problemas de conexión afectaron también a otros participantes de zonas urbanas, incluido el coordinador del taller, lo cual provocó que algunas sesiones no fueran video grabadas completas.

Falta de confianza en la participación. Si bien favoreció el que la mayor parte del grupo fueran lectores en el tema de la participación, también se destaca que esto provocó que el $29.41 \%$ de los participantes correspondiente a lectores utilitarios, no se sintiera en la confianza de externar sus opiniones por sentirse en desventaja con respecto a quienes tenían un mejor desempeño en la lectura. Además, un $11.77 \%$ manifestó que en un principio no se sentía en la confianza pero después sí. Esta problemática prevaleció sobre todo en las dos primeras partes del taller.

\section{RESULTADOS Y DISCUSIÓN}

Previo a la finalización del taller se llevaron a efecto las entrevistas a los 17 participantes. Esta se compuso de 13 preguntas correspondientes a los objetivos del taller. Con esta entrevista se pudo conocer el impacto que tuvo éste en los participantes y se pudo ver en qué medida se cumplieron los objetivos, de ahí que los resultados se presenten en base a cada uno de éstos. Cabe mencionar que a cada participante se le invitó a expresarse de forma libre y con sinceridad.

\section{OBJETIVO 1. MOTIVAR A LA BÚSQUEDA DE LA LECTURA POR PLACER A PARTIR DE ESTRATEGIAS LÚDICAS QUE PERMITAN RECONOCER SUS BENEFICIOS RECREATIVOS Y SOCIALES.}

Para estimular la lectura por placer se utilizaron dos videos motivacionales, uno titulado "leer es resistir" de Benito Taibo y otro "cómo apasionarte por la literatura" de Dardon (2020). Un lector utilitario expresó: "te hacen ver la lectura con otra cara, me llaman la atención, me emocionan hasta cierto sentido de quererme aventurar". De igual forma se utilizaron estrategias lúdicas como la lectura dramatizada, particularmente en los textos de El Campamento de Luis Pescetti y Hamlet. Otro lector utilitario comentando este último señaló: "la vez que hicimos... como esta función de que todos participáramos en personajes, me gustó el contexto en que se dio el diálogo entre todos, la lectura me causó más intriga, conocer el hecho. No lo he podido realizar [leer], pero creo que ese fue el que más me llamó la atención". Además se preguntó si los recursos utilizados en el taller habían interesado o provocado alguna reacción. En la siguiente gráfica se muestran los recursos que tuvieron más impacto en el grupo de intervención, considerando alusiones a los mismos de modo individual. Esto indica que un participante pudo referir más de uno. 


\title{
Figura 1
}

Sobre los recursos utilizados en el taller que más impactaron en los participantes

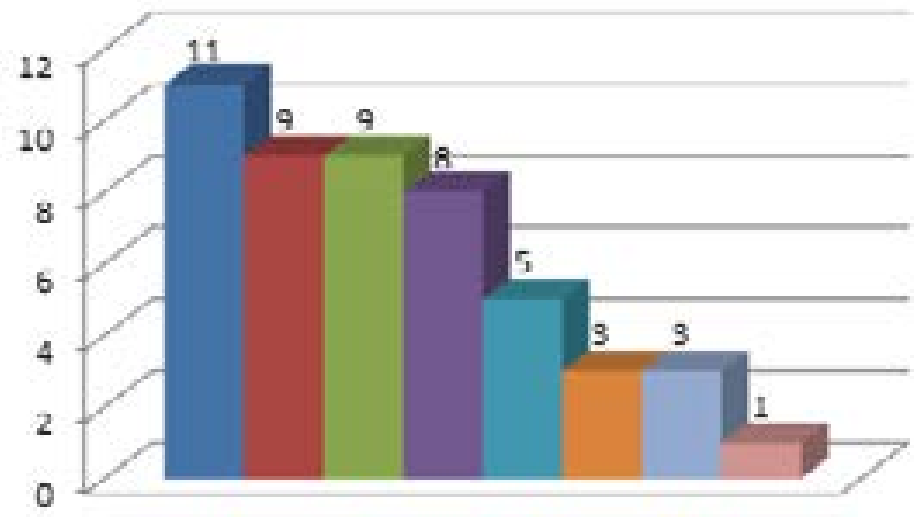

\author{
EVideo de Benito Taibo \\ - Lectura dramatizado de Hamiet \\ = Lectura gratuita \\ Video de Andrea Dardon \\ apoemas \\ II Cortometrajc \\ Experiencies de lectura \\ El banquete de Platón
}

Nota. En esta gráfica se pudo constatar que el grupo intervenido valoró positivamente los recursos que salieron de la estandarización académica, particularmente los que tuvieron puntaje más alto. Esto porque los videos presentaron la experiencia de la lectura más allá de lo escolar y los tipos de lectura (dramatizada y gratuita), implicaron un ejercicio lúdico que impulsó la participación de los estudiantes. De esta manera se pudo confirmar que la parte motivacional del taller fue pertinente para desvanecer el esquema de la lectura escolar.

\section{OBJETIVO 2. PROMOVER EL DESARROLLO DEL PENSAMIENTO ESPECULATIVO A PARTIR DE TEXTOS LITERARIOS CON TEMAS EXISTENCIALES QUE FAVOREZCAN LA REFLEXIÓN PERSONAL ESCRITA U ORAL.}

Para conocer el logro de este objetivo se analizaron dos puntos: el primero tuvo que ver con una de las condiciones para favorecer la reflexión personal, esta es la de realizarla a conciencia, es decir, por sí mismo y no por mero cumplimiento. El segundo punto se centra en determinar en qué medida los dos textos que se utilizaron para la elaboración de bitácoras (El banquete y Hamlet) y el ensayo filosófico, inspiraron una reflexión personal.

Respecto al primer punto se destaca que se solicitó a los participantes realizar las bitácoras no como una tarea académica sino como un instrumento de apoyo para el ejercicio especulativo. Estas no fueron obligatorias lo cual se constata en que no todos las entregaron, aunque esto no significa que no las hayan realizado y ocupado durante las sesiones, pues se ha de tener presente que se fueron realizando y comentando gradualmente. En la entrevista algunos participantes manifestaron realizar alguna de las dos por cumplimiento. Otros hicieron ambas a conciencia y finalmente quienes las hicieron por compromiso, aunque el porcentaje de estos últimos es bajo como se observa a continuación. 


\section{Figura 2}

Forma de realizar las bitácoras

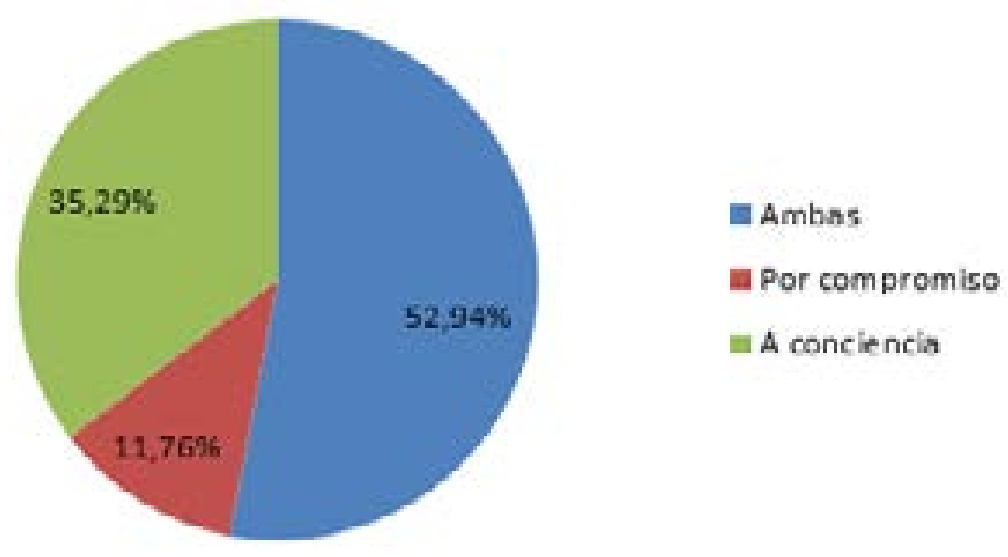

Nota. Resulto sıgniııcatıvo, segun ıa grarıca, saper que aıgunos partıcıpantes nıcıeron una de las dos bitácoras por cumplimiento. En la entrevista se destacó que fue la bitácora que correspondía a la obra El banquete de Platón, lo cual también estuvo en sintonía con lo presentado en la figura 1 , donde se refiere que tal texto fue el de menor impacto.

En lo correspondiente al segundo punto se señala que 13 participantes manifestaron haberse inspirado en los textos para realizar sus reflexiones. Algunos de ellos escribieron frases encontradas en el texto utilizado para la elaboración de la bitácora, pero su reflexión se complementó con sus propios conocimientos u otras fuentes. Por otro lado, 4 participantes señalan sólo haberse apoyado en el tema dado en la bitácora, pero con una reflexión completamente independiente del texto literario por lo cual se consideran no inspirados en éste.

Figura 3

Sobre las reflexiones personales que se inspiraron en los textos

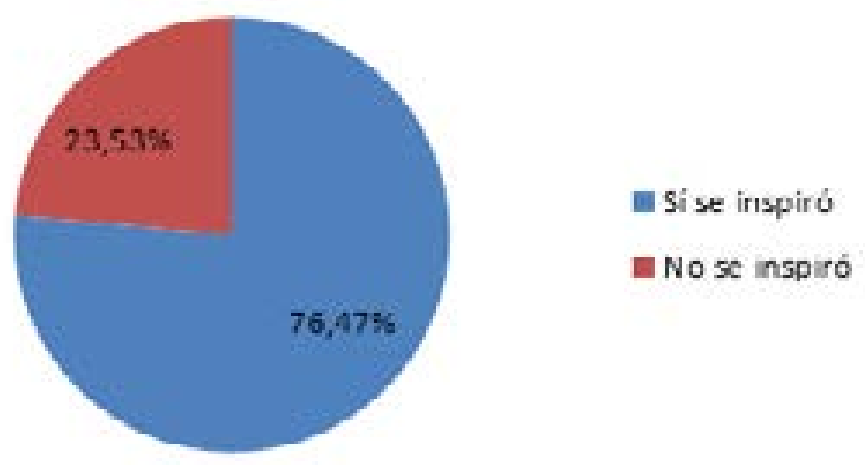

Nota. La figura mostró que en su mayoria los participantes pudieron retomar algunas ideas significativas encontradas en los textos para realizar sus reflexiones personales. El porcentaje minoritario correspondió a quienes hicieron una reflexión desvinculada del texto. 
Respecto al ensayo filosófico se pidió a los participantes que eligieran un tema existencial contenido en alguna de las obras que leyeron en la tertulia literaria dialógica, a saber La peste y El hombre en busca de sentido. Entre los criterios que se solicitaron estuvieron retomar frases 0 fragmentos del texto para partir de estas y realizar una reflexión personal. Con ello se pretendió analizar en qué medida los participantes lograron ir más allá de una interpretación literal y alcanzar la inferencial. Otro criterio fue que el ensayo no tenía una extensión específica, por lo que cada uno determinaba hasta dónde quería profundizar en el tema y cómo quería ordenarlo. Esto permitió conocer la capacidad de los participantes para estructurar sus ideas y mantener una línea temática a lo largo de su escrito, así como para vislumbrar si éste reflejaba un proceso elaborativo más allá del mero cumplimiento. Por último, se destaca que el ensayo fue evaluado, además del coordinador del taller, por dos profesores ajenos al mismo y especialistas en el área de filosofía, con el fin de constatar el nivel de reflexión alcanzado, en base a una rúbrica previamente diseñada. Los criterios evaluativos fueron específicamente los relacionados con el contenido del texto, por lo que algunos criterios académicos como la ortografía, el citado, la presentación, etc., no fueron la prioridad en la ponderación. Los criterios evaluativos fueron: estructura, interpretación, líneas argumentativas y línea temática, valorándose en cuatro categorías (excelente, bueno, mínimamente satisfactorio y deficiente).

En cuanto a los resultados obtenidos se destaca que de los 17 participantes, sólo 13 entregaron su ensayo. La extensión de los mismos fue entre 3 y 4 cuartillas. Los participantes faltantes en la entrega de su ensayo fueron dos lectores autónomos y dos utilitarios. A continuación se presentan los resultados evaluativos de los mismos en base a la rúbrica, teniendo presente que el máximo puntaje para cada rubro era de tres.

Figura 4

Resultados de la evaluación del ensayo en base a la rúbrica

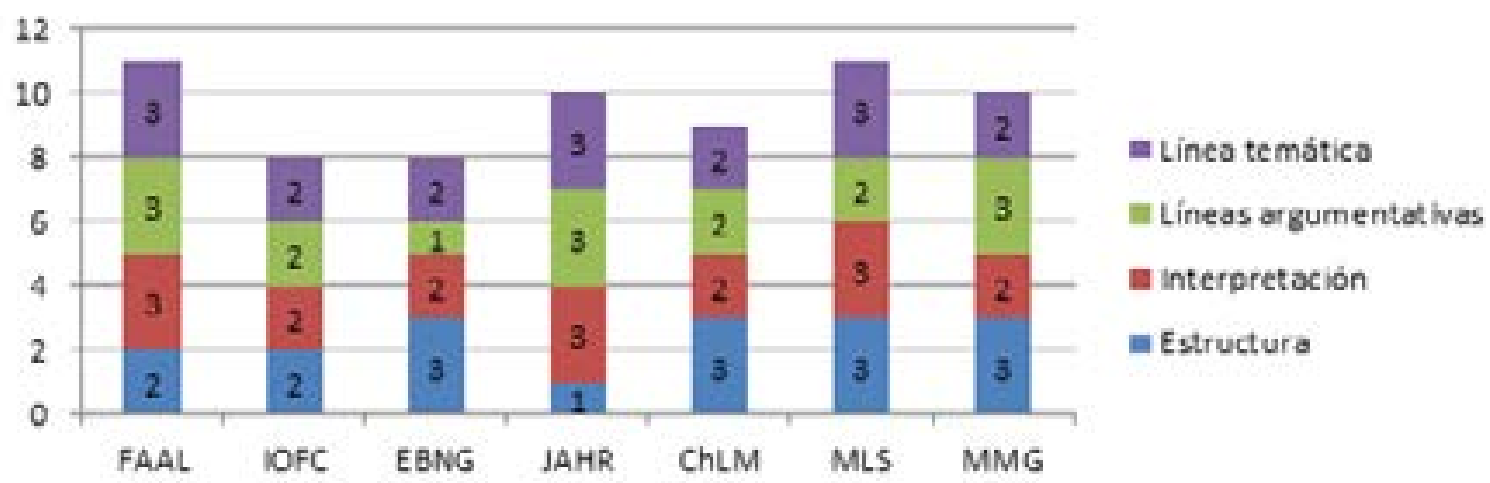




\section{Figura 5}

Resultados de la evaluación del ensayo en base a la rúbrica

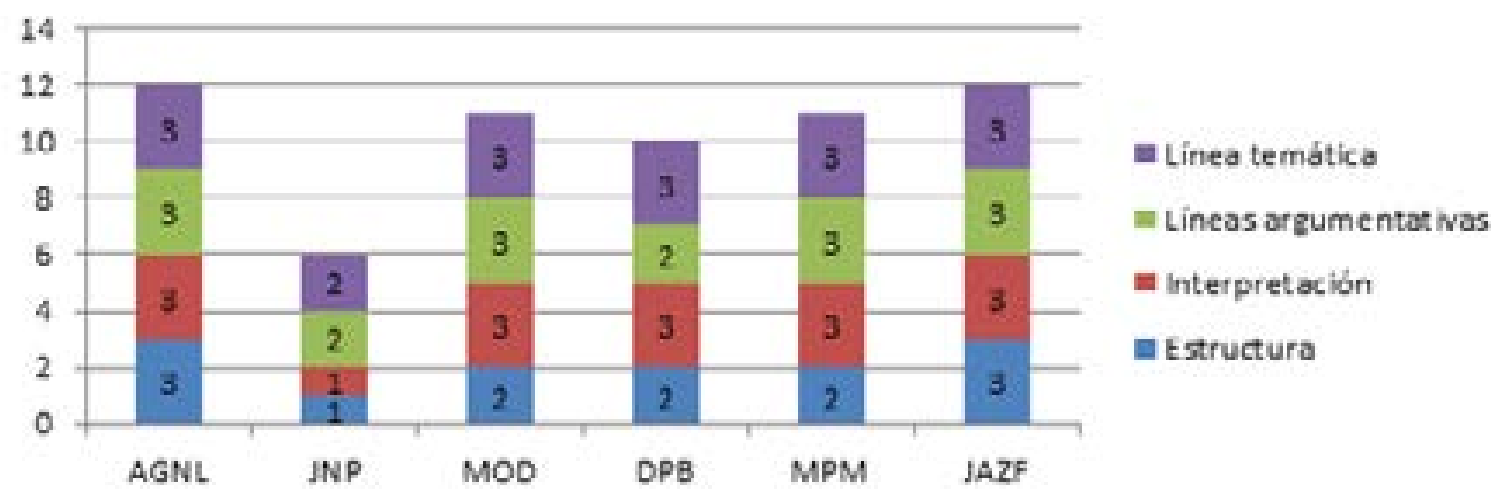

Como se puede constatar en cada una de las gráficas la mayoría de los participantes obtuvo un nivel óptimo en cada uno de los rubros, situándose entre lo bueno, equivalente a 2 puntos y lo excelente correspondiente a 3 . Respecto al rubro de interpretación, 8 participantes alcanzaron un nivel excelente, lo que implicaba ser capaz de vincular la interpretación literal con la inferencial y extraer reflexiones con cierto grado de originalidad e intertextualidad. Otros 4 participantes estuvieron en la categoría de "Bueno", lo que implicó que fueron capaces de hacer además de una interpretación literal una inferencial, pero con reflexiones poco profundizadas. Sólo un participante no logró alcanzar un nivel óptimo en la interpretación. No obstante, a pesar de ello, se cumplió el objetivo general, el cual tenía como uno de sus fines disponer a los participantes al quehacer filosófico, el cual implica ser capaz de trascender lo literal de un texto y realizar inferencias. Al respecto se presentan tres comentarios de los evaluadores a tres participantes representativos de cada tipo de lector. El primero corresponde al ensayo de JAHR (lector utilitario) del cual se refiere lo siguiente: "se aprecian las líneas argumentativas unidas al núcleo temático presentado desde inicio. Se le reconoce al autor hacer hablar el texto en su mundo por medio del sufrimiento. Se echa de menos una estructura más integral". El segundo comenta el ensayo de AGNL (lector autónomo) diciendo: "Aunque hay algunos errores ortográficos o sintácticos, el texto redactado por el estudiante es claro en su idea central, y logra plantear un problema a partir de la lectura que cita oportunamente. Además, logra traer el tema presentado al contexto actual de la pandemia. Cabe decir, por último, que aunque su trabajo no explicita una introducción y desarrollo de manera evidente, sí quedan de manifiesto estos apartados dentro de la propia lectura". Finalmente, se presenta un comentario al ensayo de JAZF (lector letrado): "El estudiante ofrece un texto bien escrito y con un estilo propio que no demerita en la exposición de sus argumentos; además retoma citas de autores y las incorpora de manera eficaz a su argumento central acerca del miedo y la ignorancia".

De todo esto se puede concluir que en cada participante hubo un esfuerzo reflexivo reflejado en su escrito y reconocido por los evaluadores. También se constata que hay una estrecha relación entre lectura y escritura, de manera que quien posee un nivel de grado superior en la primera, tiene más elementos para plasmar sus ideas y estructurarlas. Este hecho está en concordancia con lo que Garrido (2012) refiere de los alumnos con mayor nivel 
de lectura y escritura, de quienes dice que "[...] son capaces de escribir textos más amplios, claros, coherentes y mejor informados, [...]" (p. 23). Por último se muestra que el factor de la asistencia al taller influyó en el desempeño de los participantes, pues el participante que tuvo el nivel más bajo en el ensayo, fue uno de los que más inasistencias alcanzó.

\section{OBJETIVO 3. SUSCITAR EL INTERÉS POR LOS CONTENIDOS FILOSÓFICOS, A TRAVÉS DE LA LECTURA DE TEXTOS LITERARIOS CON TEMAS EXISTENCIALES QUE FAVOREZCAN LA RELACIÓN ENTRE FILOSOFÍA Y LITERATURA.}

Los resultados que corroboran este objetivo se extrajeron con dos preguntas, la primera se enfoca a conocer si el participante pudo constatar una relación del taller con sus estudios de filosofía; la segunda pretende conocer si el taller incentivó el interés por tal ciencia. Respecto a la primera, un participante (lector autónomo) señaló: "en ser más analítico, detenerme en el texto a la hora de leer porque a veces sólo leo por entregar la tarea..., pero este taller me ha brindado esa capacidad de detenimiento y de observación... traigo a colación lo de las bitácoras porque me sirven mucho en los reportes de lectura para poner realmente mi punto de vista sincero de cada lectura". También se destaca otro comentario sobre cómo el taller motivó a interesarse por la filosofía, al respecto un lector utilitario refirió: "hasta cierto punto sí, me ha ayudado a interesarme porque hay veces que los diálogos que la filosofía ofrece son un poco extensos y se necesita mucha comprensión y este taller me va ayudando a profundizar más en el sentido filosófico... El banquete de Platón me interesó mucho y despertó el leer otros diálogos o leer algunos fragmentos de San Agustín".

Figura 6

Identificación de una relación del taller con los estudios de filosofía

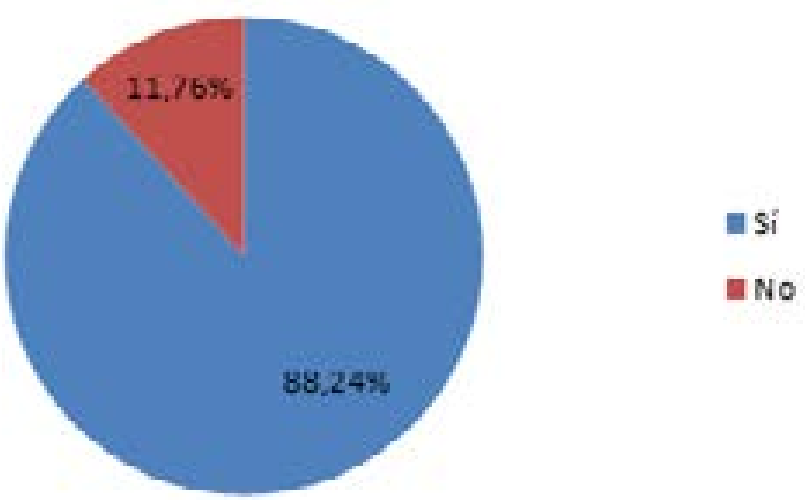




\section{Figura 7}

Sobre la motivación para interesarse por la filosofía

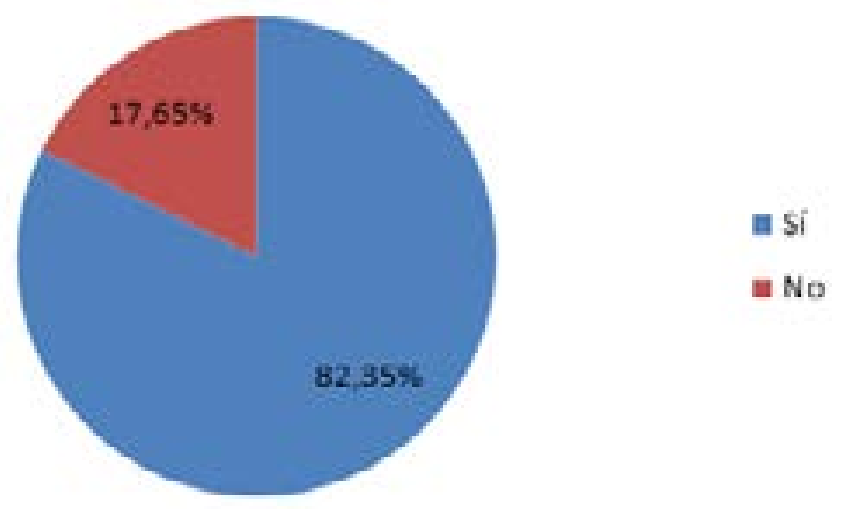

Nota. La grafica mostró que la mayoría de los participantes pudo interesarse por la filosofía a raíz del taller. El $17.65 \%$ que señaló no interesarse a partir de éste, tampoco significó que tuviese una falta de interés por tal ciencia. Sólo se quiso indicar que el taller no fue el medio para interesarse por ella.

\section{OBJETIVO 4. COADYUVAR EN LA}

PRÁCTICA DE LA LECTURA MEDIANTE EL APOYO DE UNA TERTULIA LITERARIA

DIALÓGICA QUE IMPULSE EL GUSTO POR LA MISMA Y FAVOREZCA SU PERSISTENCIA.

Para conocer en qué medida se cumplió este objetivo se preguntó a los participantes si consideraban que la tertulia literaria dialógica les había ayudado a comprender el texto elegido en su grupo. La respuesta fue afirmativa y unánime. Los participantes señalaron que mediante la tertulia pudieron conocer los puntos de vista de sus compañeros y ampliar la comprensión del contenido del texto, pues algunos se centraban en elementos de éste que para otros pasaban desapercibidos.

También se cuestionó sobre la forma en que leyeron el libro utilizado en la tertulia, reduciéndose básicamente a dos puntos: si

\section{Figura 8}

Sobre la forma de realizar la lectura de los textos compartidos en la tertulia

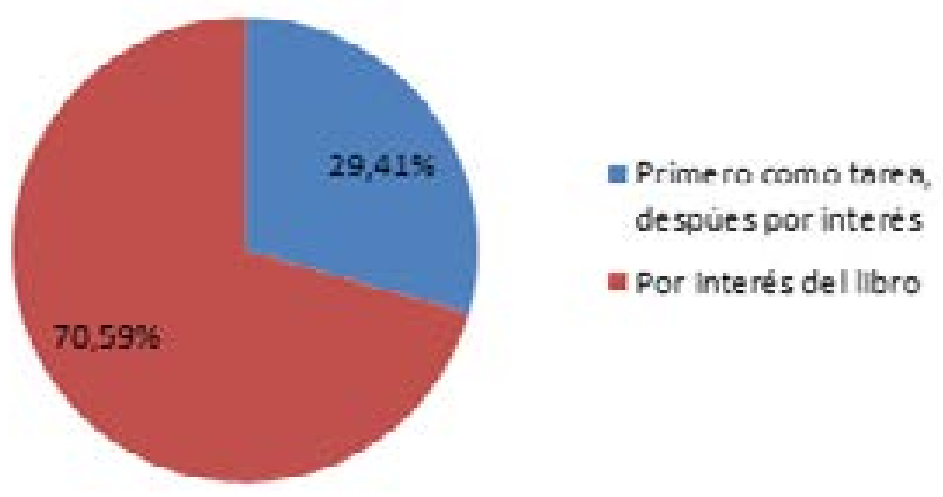


Otro aspecto que favoreció la tertulia fue el de la confianza en la participación de los lectores utilitarios quienes habían manifestado no sentirse confiados a compartir sus opiniones en la reunión general, pero que en la tertulia dado que fue un grupo más reducido y formado en su mayoría por lectores utilitarios, sintieron un clima holgado y propicio para expresarse. Esto se constató en el incremento del $11.77 \%$ correspondiente a quienes no se sentían en un principio en la confianza de compartir y después sí. En este sentido se puede concluir que el ambiente participativo del taller fue gradual y que la tertulia literaria dialógica representó un apoyo importante para dirimir los obstáculos tenidos a lo largo del mismo.

\section{CONCLUSIONES}

La implementación de un taller de promoción de lectura y estímulo de la actividad filosófica en modalidad virtual y en un ambiente educativo, da oportunidad para establecer algunas conclusiones relacionadas con la metodología y la escolarización.

Primeramente, permite reafirmar la idea de que la metodología de un taller en modalidad virtual ha de partir del contexto de los participantes. Ha de considerar el ámbito geográfico y la condición socio-económica de éstos, pues no todos están en las mismas condiciones para tomar sesiones virtuales. En las zonas indígenas y rurales las fallas de conexión son frecuentes y los talleristas poseen dificultades para participar en las actividades o hacer uso de las funciones de una plataforma de modo eficaz. Así pues, considero que mientras exista desigualdad en el acceso a servicios tecnológicos, la enseñanza virtual y lo vinculado a ella tendrá que adoptar como uno de sus principios la flexibilidad en cuanto a la metodología, los instrumentos y la integración de evidencias.
Por otro lado, se reafirma también que la promoción de la lectura en un ambiente educativo exige necesariamente promover el principio de libertad, como medida idónea para llevar el ejercicio de la lectura más allá de la escolarización. Esto porque, como han ratificado diversos teóricos, la escolarización se ha quedado sólo en medir la lectura y los factores vinculados a ella como la velocidad, la comprensión, la dicción, etc., dejando de lado la experiencia del lector. En este mismo sentido, Juan Mata (2008) apunta: "Necesitamos abolir las prácticas escolares que corrompen la experiencia literaria" (p. 44). De esta manera, considero que en el tema de la lectura, los ambientes educativos han de superar las estandarizaciones pedagógicas y han de dar lugar a la creación de comunidades de lectores que en un contexto de diálogo y convivencia respetuosa, desarrollen el gusto por la misma y las habilidades propias de la etapa escolar. Para tal efecto, se hace necesario potenciar los clubes de lectura, las tertulias literarias dialógicas, los círculos de lectura, etc., proponiendo más que imponiendo y respetando la libertad de los destinatarios.

Empero, esto no implica que se adolezca de un acompañamiento a los estudiantes o de una evaluación de las propuestas vinculadas a la lectura. En el primer caso, considero pertinente iniciar las propuestas de promoción de la lectura con un acompañamiento directo por quienes funjan como coordinadores o tutores de las mismas, pero en la medida que las circunstancias lo permitan y de forma gradual, considerando la etapa escolar, dar paso a un acompañamiento indirecto, el cual responsabilice más a los involucrados. Respecto a la evaluación de las propuestas, destaco que ésta es necesaria para conocer el desarrollo de las mismas, identificar las áreas de oportunidad, los aciertos y las fallas en el uso de estrategias. Sin embargo, no es conveniente que en el tema de la lectura ésta quede circunscrita al desempeño del estudiante y que sea ponderada con una calificación. Para 
ello considero más oportuno suscitar situaciones a lo largo de la propuesta de promoción de la lectura donde el participante pueda confrontarse o autoevaluarse y experimentar la necesidad de reafirmar el hábito de lectura. En este sentido, resulta valiosa la estrategia de compartir la experiencia de iniciación en la lectura por quienes tengan un avance significativo en ella, debido a que puede inspirar el gusto por la misma. Por lo tanto, se sostiene que escolaridad y promoción de la lectura pueden ser compatibles si la primera no absorbe a la segunda, es decir, si se evita que un taller, curso o programa de lectura se convierta en una asignatura académica. En otras palabras, siguiendo a Garrido (2012), considero que, después de la familia, la escuela es el segundo lugar idóneo para formar lectores.

\section{REFERENCIAS}

Argüelles, J. D. (2015). Por una universidad lectora. En E. M. Ramírez Leyva (coord.). Tendencias de la lectura en la universidad (pp. 15-29). Universidad Nacional Autónoma de México. http:// creativecommons.org/licenses/by-ncsa/3.0/deed.es MX

Camus, A. (2019). La Peste. Editores Mexicanos Unidos.

Cárdenas Guzmán, G. (2018). Los retos de la lectura en la era del internet. ¿Cómo ves? 20(233), 8-13.

Carlino, P. (2005) Escribir, leer y aprender en la universidad. Una introducción a la alfabetización académica. Fondo de Cultura Económica.

Carretero, M. (1993). Constructivismo y educación. Aique.

Cassany, D. y Morales, O. A. (2008). Leer y escribir en la universidad: hacia la lectura y la escritura crítica de géneros científicos. Revista Memoralia, 5(2), 6982.

Cortazar, J. (2008). Rayuela. Cátedra.

Dardón, A. (2020, 21 de marzo). "Cómo apasionarte por la literatura" [Video]. You tube. https://youtu.be/YbdTQklQn-E

Frankl, V. (1991). El hombre en busca de sentido. Herder.

Garrido, F. (2004). Para leerte mejor. Mecanismos de la lectura y de la formación de lectores capaces de escribir. Paidós.

Garrido, F. (2012). Manual del buen promotor. Una guía para promover la lectura y la escritura. CONACULTA.

Grondin, J. (2008). ¿Qué es la hermenéutica? Herder.

Guardiola, E. y Baños, J. E. (2018). Lo que ocurre cuando leemos un libro (y su efecto en nuestra vida). Rev Med Cine [Internet]. 14(1), 1-3.

Hernández Sampieri, R. y Mendoza Torres, Ch. P. (2018). Metodología de la investigación: las rutas cuantitativa, cualitativa y mixta. Mc Graw Hill.

INEGI (2020). Principales resultados. Módulo sobre lectura. https://www.inegi.org. $\mathrm{mx} /$ contenidos/programas/molec/doc/ resultados molec feb20.pdf

Instituto Nacional para la Evaluación de la Educación. (2020). Resultados nacionales 2017. Plan Nacional para la Evaluación de los Aprendizajes. http:// planea.sep.gob.mx/ms/

Mata, J. (2008). 10 ideas clave. Animación a la lectura. Hacer de la lectura una práctica feliz, trascendente y deseable. Graó. 
Morón Arteaga, N. A. (2015). Analfabetismo funcional presente en la comprensión lectora de estudiantes universitarios. CIENCIAMATRIA, 1(1), 57-71.

https://www.cienciamatriarevista.org.ve/ index.php/cm/article/view/62

OCDE (2019). PISA 2018-Resultados. https://www.oecd.org/pisa/publications/

PISA2018_CN_MEX_Spanish.pdf

Platón. (1998). Diálogos. Gredos.

Pulido, C. y Zepa, B. (2010). La interpretación interactiva de los textos a través de las tertulias literarias dialógicas. Revista signos, 43, 295-309. https://scielo. conicyt.cl/scielo.php?pid=S071809342010000400003\&script=sci arttext\&tlng=en

Sardi, V. (2014). Lecturas fronterizas: jóvenes y prácticas de lectura. En Moreno, H., García, S. y Sardi, V. Lectores, libros, lecturas. Cambios en las prácticas y hábitos de lectura (65-84). Secretaría de Cultura de la Nación (Nuestra cultura). http://www.memoria.fahce.unlp.edu.ar/ libros/pm.692/pm.692.pdf

Shakespeare, W. (2006). Hamlet y Macbeth. Universidad Veracruzana

Sisto, V. (2015). Bajtin y lo Social: Del discurso a la actividad dialógica heteroglósica. Athenea Digital, 15(1), 3-29.

https://www.raco.cat/index.php/ Athenea/article/view/292073/380577

Taibo, B. (2020, 5 de octubre). "Leer es resistir". [Video]. You tube. https://www.youtube. com/watch?v=iTEz1yOmepQ\&t=603s 